\title{
USO DE INSTRUMENTOS DE COLETA DE DADOS EM PESQUISA QUALITATIVA: UM ESTUDO EM PRODUÇÕES CIENTIIFICAS DE TURISMO
}

\author{
THE USE OF DATA COLLECTION INSTRUMENTS IN QUALITATIVE RESEARCH: A STUDY OF \\ TOURISM RESEARCH PAPERS
}

\section{EL USO DE INSTRUMENTOS DE RECOLECCIÓN DE DATOS EN UNA INVESTIGACIÓN CUALITATIVA: UN ESTUDIO EN PRODUCCIONES CIENTÍFICAS DE TURISMO}

Wagner Fernandes Costa

Professor EBTT do Instituto Federal do Rio Grande do Norte Mestrando em Turismo na Universidade Federal do Rio Grande do Norte wagnerfcosta@hotmail.com

Ana Luiza de Albuquerque Tito Mestranda em Turismo na Universidade Federal do Rio Grande do Norte analuiza.tito@hotmail.com

Paula Normandia Moreira Brumatti Professora EBTT do Instituto Federal do Rio Grande do Norte Doutoranda em Turismo na Universidade Federal do Rio Grande do Norte paulanmb@yahoo.com.br

Mauro Lemuel de Oliveira Alexandre Docente do PPGTUR/UFRN Doutor em Ciências em Engenharia de Produção (COPPE/UFRJ) mauro_alx@yahoo.com.br

Data de Submissão: 24/10/2016 Data de Aceitação: 04/10/2017

\begin{abstract}
RESUMO: Os avanços dos estudos acadêmicos em turismo, de origem multidisciplinar, têm revelado novas possibilidades epistemológicas, teóricas e metodológicas para a consolidação deste campo de pesquisas. Nesse contexto, pretende-se apresentar algumas peculiaridades e tendências das pesquisas qualitativas nos estudos do turismo. $O$ objetivo deste artigo é analisar o uso de instrumentos de coleta de dados nas produções acadêmicas, concluídas entre os anos 2010 e 2015, do programa de pós-graduação em turismo da UFRN. A análise de setenta dissertações de mestrado disponíveis nos repositórios da Instituição contribuiu para refletir sobre os caminhos metodológicos adotados pelos pesquisadores desse programa. Identificou-se uma preferência pela abordagem qualitativa, que se utiliza de diversos instrumentos de coleta de dados, como: as entrevistas semiestruturadas, os documentos provedores de dados secundários e as observações realizadas pelos pesquisadores sobre seu objeto de análise. Acredita-se que a escolha destes instrumentos tem colaborado para compreender mais profundamente o fenômeno turístico e garantir maior rigor metodológico neste campo de estudos. A ampliação desta análise requer novas investigações sobre as produções em programas de pós-graduação no Brasil, bem como reflexões acerca da adequação entre os instrumentos de coleta de dados, objetivos e métodos de pesquisas propostos pelos estudiosos do Turismo.
\end{abstract}

PALAVRAS-CHAVE: Turismo; Pesquisa qualitativa; Instrumentos de coleta.

ABSTRACT: Advances in academic studies in tourism, as a multidisciplinary field of knowledge, have revealed new epistemological, theoretical and methodological possibilities for the consolidation of this field of research. In this context, this study presents some peculiarities and trends in qualitative research in this field. This article analyzes the use of data collection instruments in academic papers in tourism completed between 2010 and 2015 for the graduate program in tourism of the Federal University of Rio Grande do Norte (UFRN). An analysis of seventy master's degree 
ISSN: 1983-7151

dissertations, available in the Institution's repositories, contributed to reflection on the methodological routes taken by the researchers of this program. A preference for the qualitative approach was identified, with the use of a range of data collection instruments, such as semi-structured interviews, documents providing secondary data, and the researchers' observations. It is believed that the choice of these instruments has furthered a deeper understanding of the tourism phenomenon, ensuring greater methodological rigor in this field of studies. The expansion of this analysis requires new research on papers of other graduate programs in Brazil, as well as more discussions about the adequacy of data collection instruments, objectives, and research methods proposed by academics in the field of tourism.

KEYWORDS: Tourism; Qualitative research; Data instruments.

RESUMEN: Los avances de los estudios académicos de origen multidisciplinario en turismo han revelado nuevas posibilidades epistemológicas, teóricas y metodológicas para la consolidación de este campo de estudios. En este contexto, se pretende presentar algunas peculiaridades y tendencias de las investigaciones cualitativas en los estudios del turismo. El objetivo de este artículo es analizar el uso de instrumentos de recolección de datos en las producciones académicas del programa de Postgrado en Turismo de la UFRN concluidas entre los años 2010 y 2015. El análisis de setenta Disertaciones de Maestría disponibles en los repositorios de la Institución contribuyó para reflexionar sobre los caminos metodológicos adoptados por los investigadores de ese programa. Se identificó una preferencia por el abordaje cualitativo, que utiliza diversos instrumentos de recolección de datos tales como las entrevistas semiestructuradas, los documentos que proporcionan datos secundarios y las observaciones realizadas por los investigadores sobre su objeto de análisis. Se cree que la elección de estos instrumentos ha colaborado para comprender más profundamente el fenómeno turístico y garantizar mayor rigor metodológico en este campo de estudios. La ampliación de este análisis requiere nuevas investigaciones sobre las producciones en programas de postgrado en Brasil, así como reflexiones acerca de la adecuación entre los instrumentos de recolección de datos, objetivos y métodos de investigación propuestos por los estudiosos del Turismo.

PALABRAS CLAVE: Turismo; Investigación cualitativa; Instrumentos de recolección.

INTRODUÇÃO

dimensão econômica que o turismo vem assumindo na atualidade,
nos mais diversos territórios, e toda a complexidade de relações que
envolve como fenômeno social têm despertado o interesse científico e exigido das pesquisas um caráter "extra-multi-disciplinar" (Tribe, 2010). Neste sentido, o crescimento dos estudos turísticos acadêmicos, nas últimas décadas, e as contribuições das mais diversas ciências revelam novas possibilidades epistemológicas, teóricas e metodológicas (Bispo, 2016). E embora o campo de conhecimento do turismo ainda seja considerado fragmentado e dotado de indefinições conceituais (Netto, Noguero e Jäger, 2011; Tribe e Liburd, 2016), novas propostas teóricas e metodológicas de análise têm sido criadas.

Apesar do turismo ser uma prática que se inicia ainda no século XIX, os estudos no campo científico somente ganham destaque após a Segunda Guerra Mundial. Em países europeus e nos Estados Unidos, os primeiros estudos em turismo estavam ligados às áreas de conhecimento já consolidadas no meio 
acadêmico como a geografia, a economia ou a administração, surgindo neste contexto os cursos superiores de hotelaria e de turismo (Barreto, 1995; Rejowski, 1996). No Brasil, a educação para a atividade turística iniciou-se na década de 1970 com o surgimento dos primeiros cursos profissionalizantes voltados para as necessidades advindas do surto desenvolvimentista e de crescimento econômico, alinhadas aos interesses do mercado turístico (Rejowski, 2013).

Atualmente, encontram-se nas universidades brasileiras as oportunidades de fortalecimento da produção do conhecimento científico em turismo com consistência metodológica. Seriam nestes ambientes que, a partir do desenvolvimento de estudos críticos, encontrar-se-iam algumas soluções para o "imbróglio teórico e prático" (Netto et al., 2011, p. 553) que permeia o turismo. A partir do estudo crítico é possível definir o método científico, o qual conduzirá à elaboração de conceitos que gerarão as observações e instigarão a análise empírica de uma realidade, confirmando ou refutando as hipóteses consistentemente (Cruz, Berberi e Guzela, 2008). Por isso, o método deve basear-se na exatidão, na objetividade e na sistematização das técnicas e dos procedimentos.

Para Marujo (2013), a multidimensionalidade do turismo permite sua análise a partir de diversas metodologias, a depender dos objetivos e do tipo de análise que se opta na investigação. Cabe ao pesquisador escolher a abordagem que responda satisfatoriamente o propósito da pesquisa. É desta maneira que as abordagens qualitativa e quantitativa podem ser apropriadas em diferentes etapas da investigação. Apesar de envolverem diferentes métodos de pesquisa, sugere que sua aplicação complementar fornece informações ao quadro geral de uma investigação, contribuindo para o seu enriquecimento.

O esforço de desvelar as particularidades, as contradições e as subjetividades que marcam a dinâmica turística induz que as pesquisas adotem, cada vez mais, uma reflexão de natureza qualitativa, atentando-se para o uso de procedimentos adequados para a interpretação das informações obtidas (Alves, 2011). Porém, na perspectiva gerencial, a análise qualitativa, por ser exploratória, adquire limitações para o processo de tomada de decisão. Ao passo que a pesquisa quantitativa, pelo universo da amostra, permite identificar tendências mais 
gerais de manifestações, consequentemente, melhor posicionamento para as decisões gerenciais (Silva, Lopes e Braga Junior, 2014).

Não se trata de priorizar o método em detrimento do conteúdo, nem de se prender à "ditadura do método", como alerta Demo (2009, p. 29), mas de reconheceraimportância dos procedimentos metodológicos paraasalvaguarda, na medida do possível, da realidade estudada. De uma forma ou de outra, a validade e o reconhecimento de um trabalho acadêmico científico passam, então, pela necessidade de clareza em relação à metodologia empregada, bem como dos instrumentos metodológicos previstos e adotados pela pesquisa, a fim de se alcançar os objetivos de análise propostos e viabilizar a reflexão sobre a realidade.

A revisão de literatura em periódicos nacionais especializados em pesquisas de turismo revela crescente preocupação sobre a produção do conhecimento científico na área, principalmente entre os anos de 2010 e 2013. Pode-se enquadrar os assuntos tratados nas publicações em: eixos temáticos das pesquisas de graduação e pós-graduação (Bastos, 2008; Santos, Possamai \& Marinho, 2009; Momm \& Santos,2010); evolução da produção/comunicação científica em turismo (Rejowski \& Aldrigui, 2007; Rejowski, 2010; Lima \& Rejowski, 2011; Santos \& Rejowski, 2013); e pertinência de abordagens metodológicas para o turismo (Costa, Lopes Júnior \& Saraiva-Lobo, 2010; Mazaro, 2011; Alves, 2011; Kovacs et al., 2012). Dentre esse último eixo, destacam-se Kovacs et al. (2011), que demonstraram haver considerável predileção por pesquisas de natureza qualitativa, de acordo com os anais do Seminário da ANPTUR - Associação Nacional de Pesquisa e PósGraduação em Turismo, analisado entre os anos de 2006 a 2008.

Nesse sentido, esse estudo se torna pertinente por apresentar algumas peculiaridades e tendências das pesquisas qualitativas no campo de estudos do turismo e refletir, a partir de um olhar crítico, sobre os caminhos metodológicos da área em questão. O objetivo, portanto, é analisar o uso dos instrumentos de coleta de dados das pesquisas qualitativas do curso de Pós-Graduação em Turismo (PPGTUR) da Universidade Federal do Rio Grande do Norte (UFRN), a partir da constatação da predileção de tal abordagem no conjunto das produções acadêmicas da área. 
A fonte de dados utilizada para a construção deste estudo correspondeu aos trabalhos concluídos no período de 2010 a 2015, no âmbito do PPGTUR, disponíveis no repositório institucional, sobre os quais se processou uma análise de conteúdo. As discussões teóricas são apresentadas em duas sessões. A primeira traz reflexões sobre a importância da produção do conhecimento em turismo; a segunda sobre as noções ligadas à abordagem qualitativa e alguns dos instrumentos de coleta de dados mais representativos no universo de análise. As demais seções contemplam aspectos metodológicos traçados, análise dos resultados e conclusão.

\section{PANORAMA DAS PESQUISAS EM TURISMO}

O campo multidisciplinar das pesquisas em turismo tem origem nas décadas de 1960 e 1970, influenciado pelos estudiosos nas áreas de Economia, Geografia, Antropologia e Sociologia. Entre os anos das décadas de 1980 e 1990, os estudos passam a adquirir abordagens filosóficas e institucionais coercitivas à visão do mercado dos negócios e da gestão do turismo (Bispo, 2016).

No Brasil, o marco para a inserção do turismo no contexto acadêmico foi criação dos primeiros cursos de graduação pela Faculdade de Turismo do Morumbi (hoje Anhembi-Morumbi), em 1971, e pela Universidade de São Paulo (USP), em 1973. O número de pessoas interessadas em se profissionalizar nesta área repercutiu na abertura de outros cursos superiores, a maioria deles concentrados no eixo Rio-São Paulo e em faculdades particulares (Rejowski, 1996; Ansarah, 2002). Já na década de 1980, decorrente de problemas econômicos no País, ocorre uma estagnação da oferta de cursos de turismo, sendo retomada em 1990 com a expansão da oferta nas capitais da região sudeste e nas demais regiões brasileiras, fato que promulgou na valorização do turismo no âmbito acadêmico (Ansarah, 2002).

Até este momento, os estudantes e os professores-pesquisadores enfrentaram dificuldades para identificar e localizar informações necessárias às primeiras pesquisas. Isso se deu por fatores variados, dentre os quais, Rejowski (1996) aponta: 1) de ordem teórico-conceitual, pela ausência de definições precisas 
e padronizadas, pela falta de terminologia consolidada e pelo aparecimento de termos estrangeiros traduzidos inadequadamente; 2) dificuldades de teor metodológico, onde se destaca a reduzida quantidade e qualidade de dados estatísticos disponíveis, bem como dificuldades ou ausência de aplicação de técnicas e instrumentos metodológicos adequados; 3) demais aspectos, como a produção de textos com incipiência científica, o descompasso entre o setor público, setor privado e instituições de ensino superior, bem como a falta de medidas confiáveis e análises objetivas para uma avaliação dos impactos do turismo sobre as instâncias sociais, econômicas, ambientais, entre outras.

Portanto, a construção das pesquisas acadêmicas do turismo no Brasil, em seus primeiros trinta anos, de 1970 a 2000, pôde assim ser caracterizada:

Constata-se que o turismo adentra o meio acadêmico, a partir do ensino privado, e aos poucos vai se configurando uma nova área de conhecimento. Por se ter desenvolvido basicamente em instituições privadas, observa-se carência de pesquisas científicas e reduzido número de pesquisadores, aliados a uma falta de estímulos ao desenvolvimento do conhecimento teórico do fenômeno do turismo neste País. (Ramos et al., 2011, p. 784).

Apesar de todo quadro de dificuldade, "a manutenção do ensino superior à luz da evolução da legislação, implicou num aprofundamento dos estudos por meio da qualificação de professores" (Silveira, Medaglia e Gândara, 2012, p.17), que contribuiu para a consolidação de um campo de pesquisa e epistemológico do turismo no país. Assim, a partir de 2000, é estabelecido um equilíbrio entre quantidade versus qualidade, surgindo propostas diferenciadas de cursos entre profissionalizantes ou acadêmicos, relacionadas ao crescimento da atividade turística brasileira e à criação do Ministério do Turismo no ano de 2003 (Ansarah, 2002; Ghiraldello e Mercuri, 2015).

Nesse contexto, também surgiram os programas de pós-graduação. Rejowski (2010) demonstrou o crescimento contínuo da produção científica entre 1994 a 2005 apresentada por instituições públicas de ensino, embora sua concentração ainda se encontrasse no eixo Rio-São Paulo. Holanda et al. (2014) identificaram que, em 2012, na área de turismo, constavam 9 cursos, dentre mestrados e doutorados em administração e turismo; turismo e hotelaria; lazer, turismo e hospitalidade. 
Atualmente, no Brasil, funcionam 16 cursos de pós-graduação stricto sensu na área de turismo, dentre eles 4 de doutorado, 8 de mestrado acadêmico e mais 4 cursos de mestrado profissional, distribuídos em instituições públicas e privadas no Distrito Federal e nos estados de Santa Catarina, São Paulo, Rio de Janeiro, Paraná, Rio Grande do Sul, Ceará, Pernambuco, Sergipe, Rio Grande do Norte $^{1}$. A oferta de cursos de pós-graduação nas regiões Nordeste e CentroOeste sinalizam um processo de descentralização importante para a produção do conhecimento na área.

Atrelado ao crescimento dos cursos de graduação e oferta dos cursos de pós-graduação (especializações, mestrado e doutorado), espera-se o crescimento das pesquisas acadêmicas na área. Para Rejowski (2013), a geração ou a produção científica, dada sua essência e sua finalidade na formação de professores e pesquisadores, têm estreita relação com a pósgraduação stricto sensu. Apesar das dificuldades encontradas inicialmente, a qualidade da produção científica em turismo nos últimos anos é reflexo do desenvolvimento dos cursos de pós-graduação e está expressa no refinamento da terminologia e dos conteúdos que colaboram para um mercado editorial qualificado (Tomelin e Ruschmann, 2013).

Pelo exposto, percebe-se um alinhamento ao pensamento de Jafari (2005), quando propõe a emergência de uma plataforma científica ao se referir à evolução da produção de conhecimento associada ao turismo. Essa sucederia toda a euforia inicial dos estudos acadêmicos, mas não acompanharia as previsões apocalípticas da plataforma de advertência. Trata-se, segundo ele, da formação de um corpo de conhecimento sobre turismo, porém pautado na produção científica.

O contexto de evolução referenciado anteriormente expressa a importância das pesquisas como um dos pilares da atividade acadêmica. Na base deste movimento de qualificação científica do turismo está a evolução da própria atividade, em seus mais diversos aspectos (social, econômico, político, ambiental,

1 Dados obtidos na Plataforma Sucupira da Fundação CAPES, do Ministério da Educação. Disponível em: https://sucupira.capes.gov.br/sucupira/public/consultas/coleta/programa/quantitativos/quantitativoles.jsf?areaAvaliacao $=27 \&$ areaConhecimento $=61300004$. Acesso em: 08 de setembro de 2017. 
entre outros), atraindo profissionais e pesquisadores interessados em adquirir conhecimentos para compreender mais profundamente o fenômeno.

Frente ao desenvolvimento da produção científica em turismo, Sakata (2002) ressalta a importância dos aspectos metodológicos nos estudos da área. "Através de métodos de pesquisa adequados, pode-se conseguir dados de pesquisa mais acurados e informações mais úteis" (op. cit., p.03) e, portanto, caberia ao pesquisador conhecer os elementos constituintes básicos da investigação científica, como a caracterização do estudo; a precisa definição do universo da pesquisa, da coleta, do tratamento e da análise dos dados. São os procedimentos metodológicos que garantem a profundidade, o rigor, a abrangência das pesquisas e os parâmetros mínimos capazes de orientar a produção de conhecimento na área.

Bispo (2016) salienta, ao desenvolver sua teoria de análise do turismo como prática, que são inúmeras as possibilidades de instrumentos de investigação na área. Os estudos utilizam-se desde técnicas de observação, entrevistas, análises documentais e descritivas, a técnicas mais específicas dos métodos etnográficos e quantitativos, embora estes últimos ainda sejam menos utilizados.

Assim, o campo do turismo de caráter multi/interdisciplinar permite uma diversidade de procedimentos metodológicos, que variam conforme a disciplina na qual o estudo se desenvolve. Uma demonstração da importância do enfoque qualitativo para as pesquisas em turismo é dada por Marujo (2013 p. 12), quando expressa que:

Segundo Cohen (1988) as contribuições mais significativas e estáveis no turismo foram feitas pelos investigadores que empregaram uma metodologia qualitativa descontínua. Muitas das suas perspectivas e quadros teóricos permitiram o ponto de partida de várias 'tradições' no estudo sociológico do turismo, o que alimentou o campo de pesquisa com uma distinta tensão intelectual, diferente dos estudos turísticos quantitativos.

Realizando o caminho necessário para o cumprimento dos objetivos, passase à discussão da pertinência e da utilização dos instrumentos de coleta de dados em pesquisas de natureza qualitativa. 


\section{PESQUISAS QUALITATIVAS E USO DE INSTRUMENTOS DE COLETA}

DE DADOS

Ao se definir uma pesquisa como qualitativa ou quantitativa, estar-se-á fazendo referência à forma como serão analisados os dados obtidos. Por outro lado, a opção de análise é um definidor do(s) instrumento(s) de coleta de dados empregado.

A pesquisa quantitativa aproxima-se da abordagem estatística na coleta, na análise e na apresentação dos dados. As formulações e as hipóteses derivadas deste método se apoiam em evidências numéricas, para o qual é preciso estudar grupos relativamente grandes de pessoas como uso de software de organização dos dados obtidos, geralmente por questionários ou formulários. A forma mais sofisticada na abordagem quantitativa faz uso de testes para verificar a pertinência dos instrumentos de coleta de dados (Veal, 2011).

Por outro lado, as pesquisas que utilizam o método qualitativo de análise pressupõem a identificação e a exploração do universo de significados que compõem o fenômeno estudado e as interações que se estabelecem, prevendo novas compreensões sobre a variedade e a profundidade dos fenômenos sociais (Bartunek e Seo, 2002 apud Terence e Escrivão Filho, 2006). O estudo dessas questões requer a aplicação de métodos claros e sistemáticos resultantes das técnicas de coleta de dados utilizadas como entrevistas, questionários, relatos, observações, entre outros.

Escolher um design de pesquisa qualitativa pressupõe uma certa visão de mundo, requer a definição como um investigador seleciona sua amostra, coleta e analisa dados e contempla assuntos como validade, confiança e ética. A pesquisa qualitativa não é linear, mas um processo de passo a passo, ou seja, um processo interativo que permite ao investigador produzir dados confiáveis e fidedignos. Assim, o processo de coleta e análise dos dados é recursivo e dinâmico, além de ser altamente intuitivo (Teixeira, 2003, p. 191).

Assim, as pesquisas qualitativas buscam compreender os mais diversos aspectos de um fenômeno, voltando-se para explicação mais detalhada de casos mais específicos, o que presume menor capacidade de generalização do que 
as de natureza quantitativa. A tendência da abordagem é recolher informações de maior intensidade, profundidade, que expresse a complexidade do objeto analisado, ou como sugere Demo (2009), possibilita uma análise verticalizada sobre poucos casos quando comparada às propostas quantitativas. Permite, portanto, conhecer com maior particularidade determinado fenômeno, sem, no entanto, ser possível elaborar generalizações.

Um estudo de abordagem qualitativa que possui como objeto um fenômeno real terá nas fontes de dados o subsídio para a composição dos textos (Eco, 2004). Portanto, a busca de informações sobre um objeto de estudo induz o pesquisador ao uso de instrumentos de coleta de dados particulares. Para Dencker (1998), aplicam-se apropriadamente a esta modalidade a observação, a entrevista e a análise documental, as quais serão discutidas a seguir.

A importância das entrevistas como instrumento de coleta de dados está ligada ao fato de que, por meio do relato verbal, apreendem-se o nível de conhecimento, as crenças, as motivações, as expectativas, os planos e as atitudes das pessoas (Selltiz, Wrightsman e Cook, 1987). Para Gil (2010), trata-se de um diálogo assimétrico em que uma das partes busca coletar informações da(s) outra(s), compreendida(as) como fontes de pesquisa.

Pelo seu nível de estruturação, as entrevistas apresentam categorias variadas. Pode-se considerar dentre as modalidades, a entrevista informal, aberta, não dirigida ou espontânea (Gil, 2010; Vergara, 2012; Goode e Hatt; 1979; Yin, 2005), cujo objetivo é eminentemente exploratório e não segue roteiro preestabelecido, ou pode ser alterado, sem prejuízo metodológico de acordo com o andamento da narrativa. O entrevistador torna-se um "catalizador completo da expressão das crenças e sentimentos do entrevistado, e do quadro de referência dentro do qual essas crenças e sentimentos assumem um significado pessoal" (Selltiz, Wrightsman e Cook, 1987, p. 43), ou seja, o entrevistado fica livre para expressar seus relatos e opiniões sobre determinado assunto, os quais serão registrados os comentários que dão a impressão de maior significação para o informante.

Na entrevista semiestruturada ou semiaberta (Vergara, 2012), o interlocutor vale-se de uma lista de tópicos ou aspectos derivados do qual o informante está envolvido, ou mesmo cuja percepção e interpretação orientam o acesso a 
outras fontes (Yin, 2005). As questões devem manter certa relação entre o tema, e o entrevistado se expressa espontaneamente sobre os pontos elencados e cujas respostas são consideradas como válidas pelo pesquisador. Goode e Hatt (1979) sugerem que se aplique um roteiro de entrevista, detentor de algumas informações sobre o entrevistado, com questões que permitam adaptações, inclusões e busca por explicações quando as repostas não forem suficientes ou adequadas.

Designa-se deentrevista estruturada, fechada ou formal quando sedesenvolve com o apoio de uma relação de questões predeterminadas e invariáveis quanto à ordem e à redação para todos (Selltiz, Wrightsman e Cook, 1987; Gil, 2010; Yin, 2005). Para Goode e Hatt (1979), trata-se de buscar superar, pela padronização, a variedade de respostas obtidas nas entrevistas não estruturadas, permitindo que sejam feitos estudos comparativos entre grupos e subgrupos.

É possível que a entrevista seja realizada a um grupo específico, chamada de grupo focal (Gil, 2010; Vergara, 2005; Vergara, 2012). Sua utilização de maneira exploratória permite a compreensão de um problema a partir da formulação de hipóteses, ou identificados subsídios para a criação de instrumentos de coleta de dados. As reuniões com duração de 2 a 3 horas são conduzidas por um moderador (que pode ser o pesquisador), contando com um número de 6 a 12 participantes que devem apresentar certa homogeneidade. Estes interagem a partir de uma pergunta genérica que aos poucos será aprofundada pelo moderador até que o tratamento do assunto específico seja esgotado (Vergara, 2005).

Os documentos, escritos ou não, também são importantes fontes de dados quando seu teor e conteúdo servem para identificação, verificação e apreciação de uma análise científica. Sua utilização é indicada para responder a um problema de pesquisa, aprofundar o conhecimento sobre um problema, orientar a formulação de hipóteses ou corroborar a evidência oriunda de outra fonte (Yin, 2005; Gil, 2010).

A origem dos documentos coletados pode ser de primeira mão ou fonte primária, quando, de ordem pública ou privada, ainda não recebeu nenhum tratamento analítico. Incluem-se cartas, escritos ou diários pessoais, documentos oficiais, como memorandos, ofícios, boletins, leis, publicações e textos oficiais, fotografias, gravações, formulários, fichas, atas, entre outros. 
De outra forma, chama-se documento de segunda mão ou fonte secundária quando, de alguma forma, já foi reunido ou organizado, tais como jornais, documentários, relatórios de pesquisa, relatórios de empresas, tabelas, estatísticas, etc. Neste caso, o dado "bruto" já foi compilado e, consequentemente, o pesquisador é usuário secundário, apropriando-se da informação gerada a partir dos dados primários (Moreira, 2010; Eco, 2004). É preciso relativizar a dimensão do que se considera "primeira" ou "segunda" mão. Eco (2004) apresenta vários exemplos da aplicação desta dimensão, que depende da relação entre o objeto de estudo e a originalidade dos dados coletados.

A observação como instrumento de coleta de dados é utilizada nas ciências sociais para estudar o comportamento do fenômeno nas condições ambientais e nas circunstâncias espontâneas de sua ocorrência, com a finalidade de extrair situações que possam ser válidas como resultados de pesquisa (Selltiz, Wrightsman e Cook, 1987; Yin, 2005). Sua utilização, explica Vergara (2012), volta-se para a percepção e registro de eventos, condições físicas e comportamentos não verbais e linguísticos, possibilitando realizar a descrição de tais circunstâncias.

Existem três possibilidades predominantes de observação em pesquisas sociais. A primeira pode ser chamada de observação simples ou não estruturada (Gil, 2010; Goode \& Hatt, 1979; Vergara, 2012), que ocorre quando não há uma padronização das técnicas de registro da observação. $O$ registro dos acontecimentos ocorre de maneira não planificada e espontânea, alinhada a um caráter exploratório que sirva de base para observações estruturadas futuramente.

A segunda possibilidade é a observação sistemática, direta ou estruturada (Selltiz, Wrightsman e Cook, 1987; Yin, 2005; Vergara, 2012), que pode ocorrer no campo ou no laboratório. Pela sua proposta de controle, é requerido que se definam o conjunto de comportamento a ser observado, o momento adequado e a forma de registro dos dados obtidos, tais como protocolos, formulários, check list, vídeo, fotografia, entre outros. Percebe-se que, em se tratando de observação sistemática, a definição de o que, quando e como observar deve ser precisa, explícita e limitada, para que as garantias procedimentais sejam válidas metodologicamente. 
A observação participante corresponde a uma terceira modalidade, na qual a pessoa ou o grupo é estudado no ambiente em que o fenômeno se desenvolve naturalmente. Em geral, sem o registro sistematizado de informação, vale-se mais do processo de interação. O pesquisador assume o papel de membro do grupo em análise, mantendo-se anônimo ou não. Assim, busca garantir informações mais próximas da realidade, em formatos variados, por meio de comportamentos fiéis e inseridas num contexto no qual adquirem seu significado (Selltiz, Wrightsman e Cook, 1987; Yin, 2005; Goode e Hatt, 1979).

O exposto não encerra as possibilidades de instrumentos de pesquisa que a abordagem qualitativa apresenta, contudo se buscou revelar os mais usuais das produções ora analisadas. Igualmente reconhecem-se que a ampliação e o uso adequado de um conjunto de técnicas e suas possíveis combinações podem colaborar com a supressão das dificuldades de teor metodológico neste campo de estudos.

\section{METODOLOGIA}

A partir do objetivo proposto, este estudo apresenta uma abordagem exploratória- descritiva (Severino, 2007), pois procura captar informações sobre o uso de instrumentos de coleta de dados nas pesquisas em turismo, conduzindo a uma aproximação atenta, porém inicial sobre o assunto, valendose da observação, do registro, da análise e da interpretação das mesmas.

A pesquisa documental, realizada de julho a outubro de 2016, foi baseada na análise do conteúdo das dissertações de mestrado do programa de pósgraduação da UFRN, defendidas entre os anos de 2010 e 2015, selecionadas mediante consulta ao acervo físico e digital da universidade. Dois fatores foram fundamentais na escolha da Instituição para desenvolvimento desse estudo. Um de ordem prática, relacionada à conveniência do acesso aos autores das dissertações para sanar possíveis dúvidas no transcurso da pesquisa; o outro, de ordem metodológica, ligada à oportunidade de avaliar uma tendência dos estudos até então apresentados no programa.

O universo da pesquisa correspondeu a toda produção stricto sensu do programa. Das 76 dissertações finalizadas desde o primeiro ano de 
funcionamento, em 2010, até o ano de 2015, foram analisadas as 70 produções disponíveis no acervo institucional. A amostra foi restrita às dissertações de mestrado, uma vez que no período de coleta dos dados ainda não havia teses de doutorado concluídas. Ressalta-se que 06 dissertações foram excluídas da análise por terem sido defendidas no mesmo período da análise e não puderam ser consultadas no acervo digital e físico da UFRN. Considera-se, assim, a amostra significativa para fins metodológicos, não acarretando prejuízos quanto à relevância dos dados coletados nem ao rigor científico da análise.

A aplicação da análise de conteúdo (Bardin, 2009) das dissertações ocorreu por meio da categorização de elementos textuais que evidenciassem a escolha pela abordagem de pesquisa qualitativa e seus respectivos instrumentos de coleta de dados. A partir desse conjunto de categorias, foi possível inferir sobre as opções metodológicas dos pesquisadores. Priorizou-se, portanto, como categorias, a análise da abordagem empregada no estudo, o tipo de instrumento de coleta de dados e suas respectivas subdivisões.

Apesar de alguns resultados serem apresentados por meio de gráficos e tabelas, considera-se como prioritário o processo de interpretação. O recurso descritivo revela, a partir da intensidade do uso de determinados instrumentos de coleta, meios para a compreensão do significado destes instrumentos no processo de produção científica em turismo.

Uma vez selecionada a amostra e definidas as fontes de pesquisa, pode-se considerar que a pesquisa passou pelas seguintes etapas:

Figura 1: Fluxograma dos procedimentos de pesquisa
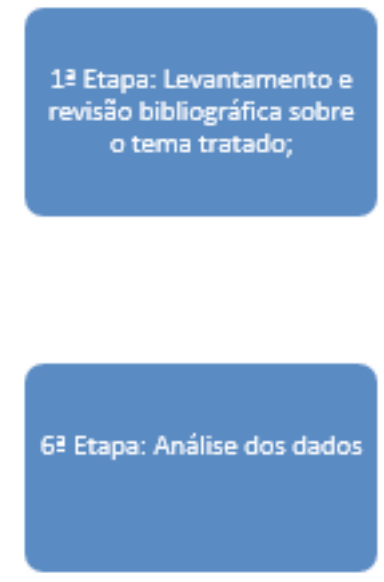

2 Etapa: Identificação dos trabalhos apresentados no âmbito do programa de pós-graduação em turismo da UFRN;

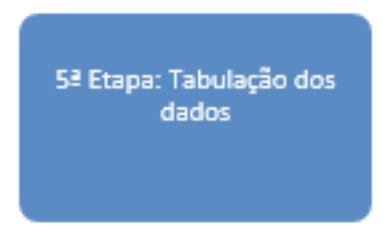

32 Etapa: Busca dos

trabalhos no acervo

institucional (virtual e

fisico);

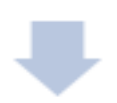

$4 a$ Etapa : Identificaçäo das pesquisas qualitativas e quali-quantitativas

Fonte: Dados da pesquisa (2016). 


\section{ANÁLISE DOS INSTRUMENTOS DE COLETA DE DADOS EM PESQUISAS DE TURISMO: USOS E ESPECIFICIDADES}

Por meio da análise das produções disponíveis para investigação, buscouse verificar inicialmente qual a abordagem utilizada, definindo-se entre três possibilidades: qualitativa, quantitativa e quali-quantitativa. Posteriormente, reconhecendo os limites traçados no objetivo deste estudo, os esforços foram concentrados na identificação dos procedimentos de coleta de dados utilizados somente nas pesquisas que se declararam qualitativas e quali-quantitativas.

Tabela 1 - Tipos de abordagem de análise empregada nas pesquisas do PPGTUR/ UFRN (2010-2015)

\begin{tabular}{ccc} 
Abordagem de análise da pesquisa & Quantidade & \% \\
\hline Quantitativa & 15 & 21,42 \\
Qualitativa & 42 & 60 \\
Quali-Quanti & 13 & 18,57 \\
Total & $\mathbf{7 0}$ & 100 \\
\hline
\end{tabular}

Fonte: Dados da pesquisa (2016).

Percebe-se uma ampla inclinação na utilização da abordagem qualitativa nas pesquisas desenvolvidas por meio do Programa de Pós-graduação em Turismo da UFRN (Tabela 1). Quando considerado o conjunto de produções com abordagem qualitativa somado ao de abordagens quali-quantitativas, obtevese um percentual superior a 78\%. Dencker (1998) alerta que o tratamento e a coleta dos dados estão ligados com o tipo de abordagem presente na pesquisa, o que torna esta informação importante, pois a opção de análise é um definidor do(s) instrumento(s) de coleta de dados mais apropriado a ser empregado. A preferência pela abordagem qualitativa conduz as pesquisas à utilização de instrumentos que não privilegiam informações numéricas, mas sim, como sugere Gil (2010), à eleição de instrumentos que evidenciam o significado ou a profundidade das informações para certos fenômenos.

Por outro lado, a presença de 13 (18,57\%) pesquisas de natureza quali-quantitativa ratifica o entendimento de que as abordagens de análise são mais complementares do que opostas. Em geral, uma precede a outra na busca completa de explicações que, para Veal (2011), conduz as pesquisas para um patamar superior de relevância. 
No Gráfico 1 apresentam-se os instrumentos de coleta de dados utilizados pelos pesquisadores. Foram considerados a partir dos objetivos traçados somente aquelas produções que apresentaram 0 uso das abordagens qualitativas e quali-quantitativas.

Gráfico 1-Instrumentos de coleta de dados empregada nas pesquisas do PPGTUR/ UFRN (2010-2015)

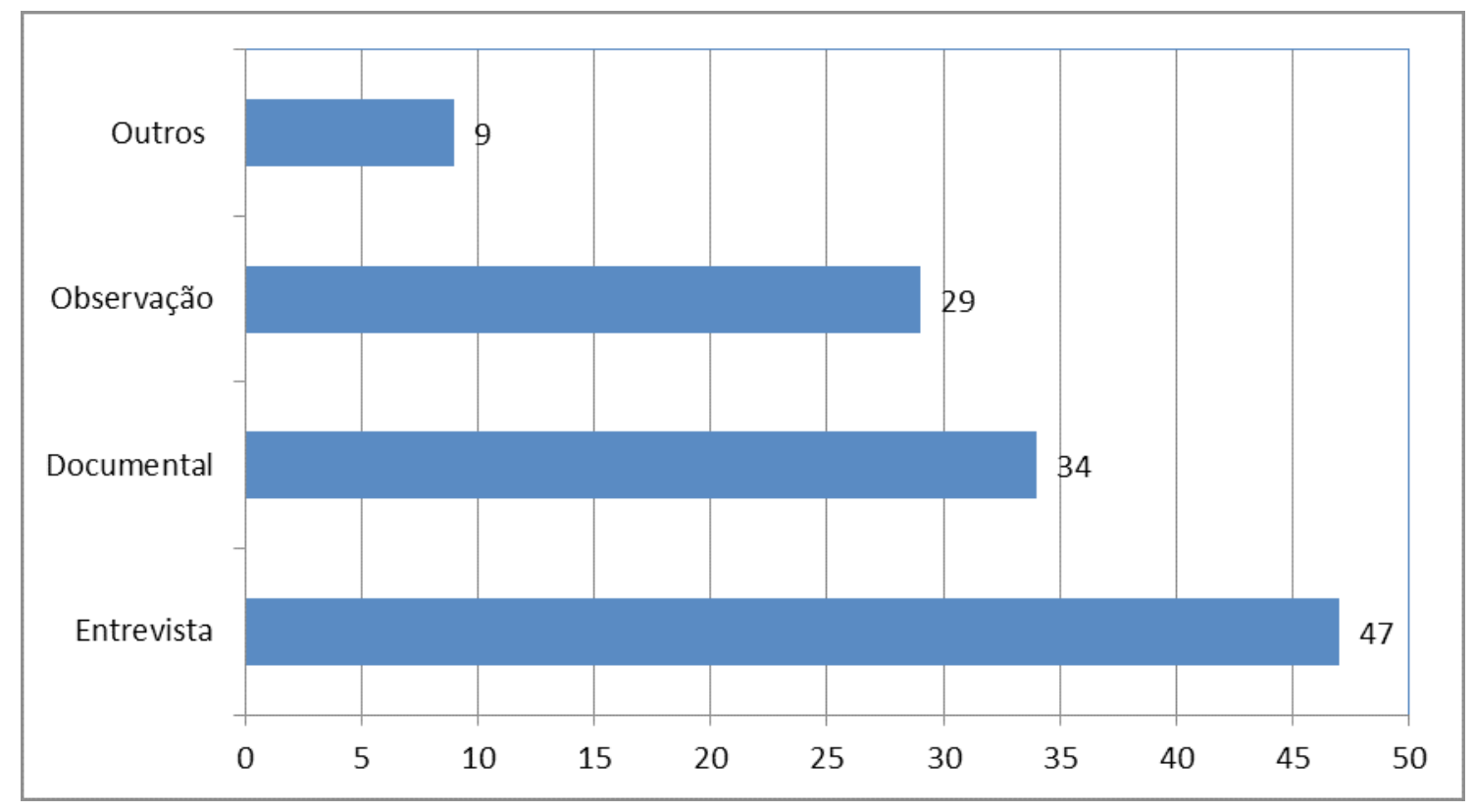

Fonte: Dados da pesquisa (2016).

Os dados revelam a importância atribuída à entrevista para a apreensão das opiniões, do conhecimento e da percepção de atores sociais diversos no âmbito das pesquisas sociais. Verifica-se que 47 (67,14\%) pesquisas recorreram a este instrumento para obter os dados necessários. Tendo como característica básica a interação social para a captação de informações de interesse, as entrevistas assumem, também nas pesquisas em turismo, a relevância e a adequação já verificadas nas pesquisas sociais (Goode e Hatt, 1979; Vergara, 2012). Em número de 34 (48\%) e 29 (41,42\%), revela-se, respectivamente, a utilização de documentos e da observação como instrumentos para a coleta dos dados dos trabalhos analisados.

Quando somados os números dos recursos de coleta de dados utilizados, obtém-se número superior à quantidade de pesquisas analisadas (70 trabalhos). Este fato ocorre pela utilização combinada dos instrumentos. Considerando que os instrumentos apresentam especificidades quanto à sua efetividade, recomenda-se que se opte pela pertinente complementaridade dos meios de coleta de dados e evidências (Yin, 2005; Goode e Hatt, 1979). 
Conforme apresentado no Gráfico 2, 22 pesquisas (40\%) combinaram três formas de instrumentos de coleta de dados (entrevistas, observação e documentação). Das demais produções analisadas, 20 (36\%) se valeram de dois instrumentos para a obtenção de dados, combinando as possibilidades de observação-entrevista, entrevistas-documentos e documentos-observação. Apenas 8 (15\%) se apoiaram em um único instrumento de coleta. Houve a ocorrência de 5 trabalhos (9\%) que utilizaram instrumentos de coleta de dados próprios de abordagem quantitativa (survey, testes) que não fazem parte do escopo de análise desta pesquisa.

Gráfico 2- Combinação de instrumentos de coleta de dados empregada nas pesquisas de mestrado do PPGTUR/UFRN (2010-2015)

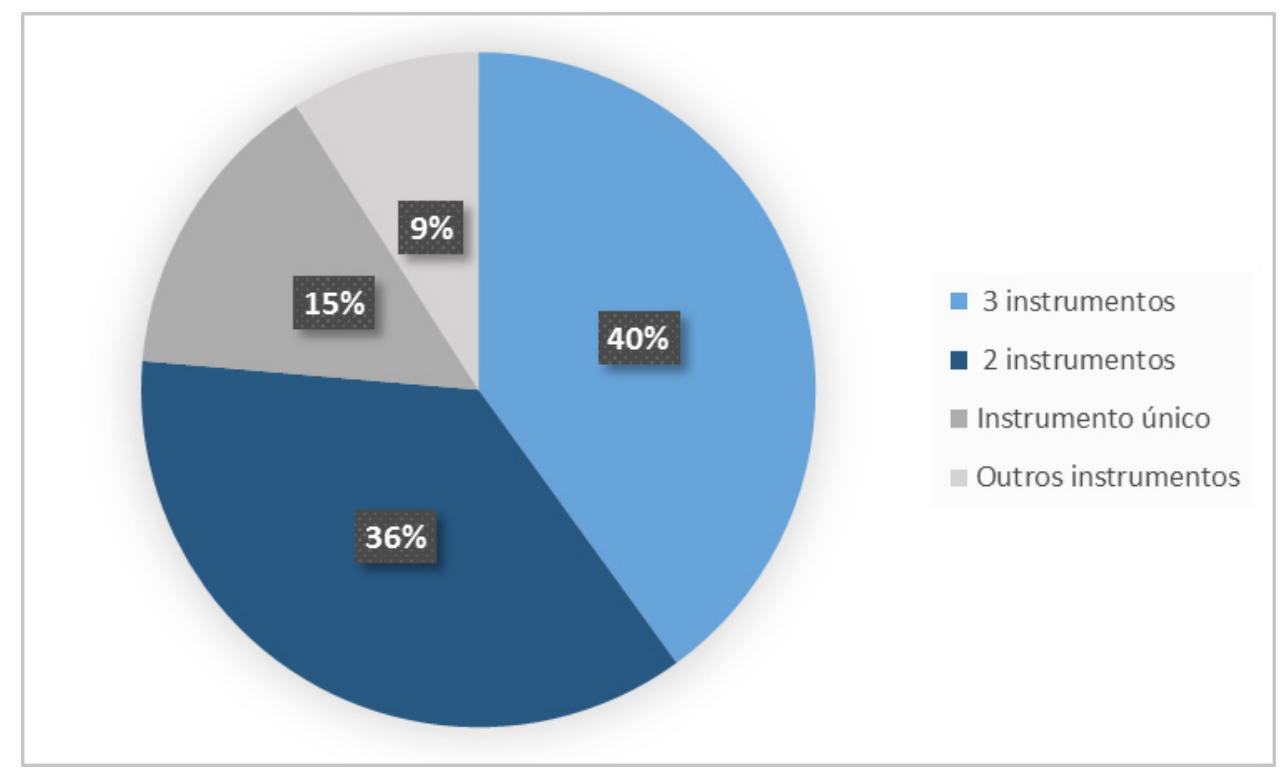

Fonte: Dados da pesquisa (2016).

O que se apreende é que a escolha e a adequação de um ou mais instrumentos de coleta estão relacionadas ao objeto de estudo. Em sua maioria, não foi suficiente a utilização de apenas um, recorrendo, o pesquisador, a dois ou mais instrumentos para busca de respostas para seu problema de pesquisa.

Os instrumentos de coleta apresentam especificidades próprias. Debruçandose sobre as características de cada um, verificou-se como se deu a utilização dos mesmos, cujos resultados são analisados na Tabela 2, 3 e 4.

Tabela 2 - Tipos de entrevistas utilizadas nas pesquisas do PPGTUR/UFRN (2010-2015).

Tipologia de entrevistas

Estruturada

Semiestruturada

\section{Quantidade}


Fonte: Dados da pesquisa (2016).

Para as entrevistas, quatro tipos de aplicação foram identificados. Como observado na Tabela 2, também houve a ocorrência de combinações entre os tipos estruturada, semiestruturada, não estruturada e focal. Como as aplicações foram feitas a diferentes atores sociais, coube, portanto, a utilização de um ou mais tipos específicos de entrevista, gerando as combinações. De forma combinada ou isoladamente, há considerável predomínio das entrevistas semiestruturadas. Das 55 pesquisas qualitativas e quali-quantitativas, 44 utilizaram este tipo de entrevista.

A preferência pelas entrevistas semiestruturadas indicam a amplitude de sua utilização na obtenção de informação sobre os mais diversos aspectos da vida social. Para Selltiz, Wrightsman e Cook (1987), este tipo possibilita a obtenção de dados com maior cobertura e complexidade quando comparado a outros, seja pela profundidade que a questão pode carregar, seja pela capacidade de superar o possível desinteresse ou dificuldade de resposta dos entrevistados. Pela forma de aplicação e pela flexibilidade na interação, torna-se possível corrigir erros de interpretação por parte dos informantes, eliminando respostas equivocadas e, consequentemente, conduzir a obtenção de dados mais fidedignos.

Destaca-se a pouca utilização das entrevistas em grupo (ou grupo focal). Apesar das vantagens no uso das entrevistas grupais (pelo intenso fluxo de informações e economia de tempo) quando comparado às entrevistas individuais (Vergara, 2012), somente 1 (uma) pesquisa utilizou este recurso, o que indica pouca preferência pelo uso deste formato entre os pesquisadores do programa de pós-graduação (PPGTUR-UFRN).

A presença de entrevista estruturada foi marcante em pesquisas qualiquantitativas, sinalizando uma tendência quanto à sua aplicação. Este fato está relacionado à composição das questões, que são marcadas por uma relação fixa de respostas, aproximando-se de um formulário, apresentando alto nível de rigidez (Yin, 2005; Gil, 2010). 
O uso de documentos representa importante recurso para as produções em turismo analisadas. A Tabela 3 expõe os tipos de documentos utilizados, verificando-se o uso combinado das diversas possibilidades. Desta forma, a soma do uso dos tipos de documentos utilizados (38) foi superior à quantidade total de trabalhos com emprego de fonte documental (34). Os dados documentais foram diversos, desde registros estatísticos a documentos públicos obtidos em instituições governamentais e não governamentais, cujas decisões e ações afetam os rumos da atividade turística de municípios e estados da federação.

Tabela 3 - Tipos de documentos utilizados nas pesquisas do PPGTUR/UFRN (2010-2015)

Tipologia de documentos

Primeira mão

Segunda mão

Total
Quantidade

14

24

38

Fonte: Dados da pesquisa (2016).

Procurou-se diferenciar documentos de primeira mão e documentos de segunda mão. Apoiado em Moreira (2010) e Eco, (2004), consideraram-se documentos de primeira mão aqueles cujo conteúdo não era proveniente de qualquer análise ou processo de compilação, mas sim se configuravam como resultado ou ato orientado de uma decisão, de deliberações ou de resultado de discussões em reuniões, tais como memorandos, ofícios, atas e registros cartoriais, bem como o conjunto de imagens em diversos formatos, resgatados de arquivos pessoais. Por outro lado, consideram-se documentos de segunda mão aqueles que, de alguma forma, são produtos de uma organização ou reflexão, tais como periódicos, documentários, relatórios de pesquisa, relatórios de empresas, fontes estatísticas censitárias, estudos institucionais, entre outros.

Observou-se uma preferência pela utilização dos documentos de segunda mão (24 trabalhos) frente à utilização de dados de primeira mão (14 trabalhos). Fontes de segunda mão são menos onerosos aos pesquisadores, pois dispensam o contato do pesquisador com os sujeitos da pesquisa por meio da consulta remota de informações já processadas. A disponibilidade virtual e regular de dados elaborados por instituições de caráter público reduz consideravelmente os custos de acesso e reprodução dos mesmos (Selltiz, Wrightsman e Cook, 1987). 
Por outro lado, como um recurso complementar à entrevista ou à observação, as fontes de segunda mão auxiliam na verificação das hipóteses desenvolvidas com informações obtidas via outro instrumento, sendo, portanto, suficientes ao pesquisador.

A observação corresponde a uma terceira preferência de instrumento para obtenção de dados nas pesquisas desenvolvidas no PPGTUR. Os resultados desta análise encontram-se na Tabela 4. A observação perpassa por todos os momentos da pesquisa, porém é no momento de coleta de dados que ganha sua condição mais importante no processo (Gil, 2010). Poucas pesquisas utilizaram a observação como recurso. Dentre as 55 produções com abordagens qualitativas, somente 29 declararam o uso da observação como instrumento de coleta de dados.

A principal vantagem da observação é a possibilidade de verificação do fenômeno de forma direta pelo pesquisador. A não interferência de intermediários para a obtenção de dados permite reduzir a subjetividade sobre o objeto da investigação. No entanto, os dados sinalizam pouca utilização deste recurso ou não são evidenciados na descrição dos procedimentos metodológicos nas dissertações.

Tabela 4 - Tipos de entrevistas utilizados nas pesquisas do PPGTUR/UFRN (2010-2015)

\section{Tipologia de observação}

Observação participante

Observação não estruturada

Observação estruturada

Total

Quantidade

8

14

7

29

Fonte: Dados da pesquisa (2016).

Dentre as 29 pesquisas que utilizaram a observação como instrumento de coleta de dados, 14 (48,27\%) optaram pela observação não estruturada. Se, por um lado, a observação pode identificar comportamentos e relações empíricas até então desconhecidas sobre o objeto de estudo, por outro, é fundamental que, como procedimento científico, atenda a um objetivo de pesquisa, sendo direcionada e planejada para manter o controle sobre sua validade e precisão. $O$ predomínio desta tipologia sugere que a observação, na maioria das pesquisas, 
é utilizada como complemento a outros instrumentos de coleta, assumindo, nestas circunstâncias, papel secundário.

Os trabalhos que utilizaram a observação participante e a observação estruturada, 8 e 7 dissertações respectivamente, demonstraram-se mais criteriosos na realização da coleta de dados.

A observação estruturada busca evitar a subjetividade presente no olhar do observador, pois está apoiada num modelo de referência, para que as impressões sejam refinadas, e relacionada a diferentes interpretações dadas ao mesmo fato observado.

Por fim, a observação participante corresponde a mais uma possibilidade de coleta de dados nas pesquisas em turismo. A crítica sobre a utilização deste recurso relaciona-se à presença declarada do pesquisador. Acreditase que este fato pode gerar alguma alteração no comportamento do ator observado, levando-o a agir de forma diferente ou a assumir uma postura "desejável", ou à supressão de posturas "negativas" e, nestas condições, o procedimento perderia parte de sua capacidade de absorção da realidade (Gil, 2010; Selltiz, Wrightsman e Cook, 1987; Goode e Hatt, 1979). Porém, sua utilização tem crescido no âmbito das pesquisas em turismo (PPGTUR - UFRN), sendo mais frequente em relação à utilização da observação estruturada. Este fato evidencia o reconhecimento da importância sobre a imersão do pesquisador no grupo, comunidade ou situação analisada, proporcionando uma maior aproximação com o objeto de pesquisa, quando comparado à observação simples ou estruturada. Diferentemente dos outros instrumentos, é possível verificar na Tabela 4 que não houve combinações dos tipos de observação.

\section{CONSIDERAÇÕES FINAIS}

O avanço das produções científicas qualificadas e validadas de pesquisa em turismo, no Brasil, passa a requerer a perspectiva da meta-análises, o estudo da própria pesquisa na busca de aprofundar-se cada vez mais. Nesse sentido, enquadra-se este estudo, uma vez que aponta a questão instrumental, seu 
uso, incidência e aplicabilidade como recurso importante para a construção do conhecimento.

Os usos de instrumentos de coleta de dados aplicados nas produções acadêmicas de Pós-Graduação em Turismo (PPGTUR -UFRN), entre 2010 e 2015, apontam uma tendência na escolha de abordagens qualitativas em relação às abordagens quantitativas de análise; demonstrando, ainda, a presença das duas abordagens de forma combinada, porém em menor proporção.

Acredita-se que este fato se relaciona ao caráter fenomenológico do turismo e à influência das ciências, como a Sociologia, a Geografia, a Antropologia e, posteriormente, a Administração, que possibilitam esta importante flexibilização metodológica. No entanto, ressalta-se que a investigação e a aplicação de novas técnicas e métodos de pesquisas são importantes para a evolução do campo de conhecimento em turismo.

Nas pesquisas qualitativas, verificou-se que o principal instrumento utilizado foram as entrevistas, preferencialmente do tipo semiestruturada, combinadas a outros modelos quando em pesquisas quanti-qualitativas.

No uso das fontes documentais, houve a predileção por dados de segunda mão, ou seja, informações anteriormente sistematizadas. No tocante ao uso da observação como instrumento de pesquisa, identificou-se uma maior frequência em sua forma não estruturada.

A análise dessas produções aponta para um uso diversificado e combinado de instrumentos de coleta de dados. Estes se relacionam diretamente aos objetivos propostos pelos pesquisadores, fato que contribui para a maior consistência nas análises do turismo como fenômeno social e para o desenvolvimento de suas teorias e campo epistemológico relacionado à realidade brasileira.

O estudo apresentou uma tendência na escolha de métodos de análises e utilização de algumas técnicas de coleta de dados nas pesquisas de turismo no PPGTUR da UFRN. Porém se sugere que, para o aprofundamento desta análise, seria importante investigar as produções de outros programas stricto sensu desenvolvidas no país, bem como refletir sobre a adequação entre os 
instrumentos de coleta de dados, objetivos e métodos de pesquisas propostos pelos pesquisadores. Entende-se que os estudos qualitativos se fundamentam numa clara definição da postura epistemológica adotada, passando pela adequada eleição dos métodos até os procedimentos de coleta e análise dos dados.

O desenvolvimento dos programas de pós-graduação e das respectivas pesquisas em turismo requer o emprego de opções metodológicas apropriadas às abordagens de análise, sejam elas quantitativas ou qualitativas. A análise dos caminhos metodológicos das produções científicas em turismo colabora para o desenvolvimento do olhar crítico sobre as produções acadêmicas e as discussões neste campo do conhecimento.

\section{REFERÊNCIAS}

Alves, M. L. B. (2011). Reflexões sobre a pesquisa qualitativa aplicada ao turismo. Turismo em Análise, 22 (3), 599-613.

Ansarah, M. G do R. Formação e capacitação do profissional em turismo e hotelaria: reflexões e cadastro das instituições educacionais no Brasil. São Paulo: Aleph, 2002.

Bardin, L. (2009). Análise de conteúdo. Lisboa: Edições 70, LDA.

Barretto, M. (1995). Manual de Iniciação ao estudo do turismo. Campinas: Papirus.

Bastos, S. (2008). A produção científica do Mestrado em Hospitalidade (2002-2008). Revista Hospitalidade, ano V, 2, 120-132.

Bispo, M. de S. (2016). Tourism as practice. Annals of Tourism Research, v.61, 170-179.

Costa, F. J., Lopes Júnior, E. P. \& Saraiva-Lobo, R. J. (2010). Métodos quantitativos em cursos de bacharelado em turismo: Uma análise da atitude e do interesse dos estudantes. Revista Turismo Visão e Ação, 12 (2), 216-229.

Cruz, G., Berberi, A. P. C. \& Guzela, M. T. (2008) Ciência e Pesquisa: reflexões sobre a inserção do turismo e do ensino superior frente ao panorama científico. Pasos- Revista de Turismo y Patrimonio Cultural, 6 (1), 109-116.

Demo, P. (2009). Metodologia do trabalho científico. São Paulo: Atlas.

Dencker, A. (1998). Pesquisa em Turismo. São Paulo: Atlas. 
Eco, U. (2004). Como se faz uma tese. 19. ed. São Paulo: Perspectiva.

Ghiraldello, L. \& Mercuri, E. N. G. da S. (2015). Integração Acadêmica de Estudantes do Ensino Superior: um estudo sobre ingressantes de um curso de turismo. Turismo em Análise, 26 (2), 403-425.

Gil. A. C (2010). Métodos e técnicas de pesquisa social. 6 ed. São Paulo: Atlas.

Goode, W. J. \& Hatt, P. K. (1979). Métodos em pesquisa social. 7.ed. São Paulo: Ed. Nacional.

Holanda, L. A., Widmer, G. M. \& Leal, S. R. (2014). A produção científica em turismo no Brasil: reflexões e proposições a partir de um estudo revisional. Anais Brasileiros de Estudos Turísticos - ABET, [S.I.], 72-79.

Jafari, J. (2005). El turismo como disciplina científica. Política y Sociedad. 42 (1), 39-56.

Kovacs, M. H., Barbosa, M. L. A., Souza, A. G. \& Mesquita, A. E.P. (2012). Pesquisa em turismo: uma avaliação das metodologias empregadas nos artigos publicados nos anais no triênio do seminário anual da associação brasileira de pesquisa e pós-graduação em turismo anptur. Revista Visão e Ação. 14 (1), 19-34.

Lima, J. R. \& Rejowski, M. (2011). Ensino superior em turismo no Brasil: a produção acadêmica de dissertações e teses (2000-2009). Revista Brasileira de Pesquisa em Turismo, 5 (03), 406-432.

Marujo, N. (2013). A pesquisa em turismo: reflexões sobre as abordagens qualitativa e quantitativa. Turydes: Revista de Investigación en Turismo y Desarrollo Local, 6 (14), 1-16.

Mazaro, R. (2011). Conocimiento científico em ciencias sociales y proposición de modelos en turismo. Turismo em Análise, 22 (03), 561-578.

Milagres, V. R. (2014). Programas de pós-graduação em turismo no Brasil: Um olhar sobre a sustentabilidade. Tese de Doutorado. Programa de Pós-Graduação em Desenvolvimento Sustentável, da Universidade de Brasília, Centro de Desenvolvimento Sustentável.

Momm, C. F. \& Santos, R. N. M. (2010). Conhecimento científico produzido nos cursos de pós-graduação (stricto sensu) em turismo e áreas correlatas no Brasil no período de 2000 a 2006. Revista Brasileira de Pesquisa em Turismo, 4 (02), 64-85.

Moreira, S.V. (2010). Análise documental como método e como técnica. In: Duarte, J.; Barros, A. (org.). Métodos e Técnicas de pesquisa em comunicação. São Paulo: Atlas.

Netto, A. P., Noguero, F. T. \& Jäger, M. (2011). Por uma visão crítica nos estudos turísticos. Turismo em Análise, 22(3), 539-560. 
Ramos, M. G. G.; Garcia, T. E. M.; Hallal, D. R. \& Müller, D. (2011). Movimentos da expansão e diversificação da Educação Superior em Turismo no Rio Grande do Sul. In: Book ofproceedings, vol. I- International Conference on tourism \& Management Studies. Algarve Portugal, 777786. Disponível em: ttp://repositorio.ufsc.br/xmlui/handle/123456789/97021. Acesso em 25 de setembro de 2017.

Rejowski, M. (1996). Turismo e pesquisa científica. Campinas-SP: Papirus.

Rejowski, M. (2010). Caracterização da produção científica sobre Turismo no Brasil - Estudo documental das teses de doutorado (1990 a 2005). Anais do VII Seminário ANPTUR. São Paulo: Universidade Anhembi Morumbi.

Rejowski, M. (2010). Produção Científica em Turismo: análise de estudos referenciais no exterior e no Brasil. Turismo em Análise, 21 (2), 224-246.

Rejowski, M. (2013). A pesquisa em turismo e hotelaria: cenário nacional. In: Ruchmann, D. Tomelin, C.A. Turismo, ensino e práticas interdisciplinares. Barueri: Manole.

Rejowski, M. \& Aldrigui, M. (2007). Periódicos científicos em turismo no Brasil: dos boletins técnico-informativos às revistas cientificas eletrônicas. Turismo em Análise, 18 (02), 245-268.

Ruschmann, D. Tomelin, C.A. (2013). Turismo, ensino e práticas interdisciplinares. Barueri: Manole.

Sakata, M.C.G. (2002). Tendências metodológicas da pesquisa acadêmica em turismo. Dissertação de Mestrado em Ciências da Comunicação, Turismo e Lazer da Escola de Comunicações e Artes da Universidade de São Paulo.

Santos, G. E. de O. \& Rejowski, M. (2013). Comunicação científica em turismo no Brasil: Análises descritivas de periódicos nacionais entre 1990 e 2012. Revista Brasileira de Pesquisa em Turismo, 7 (1),149-167.

Santos, M. M. C., Possamai, A. M. D. P. \& Marinho, M. F. (2009). Pesquisa em turismo: panorama das teses de doutorado produzidas no Brasil de 2005 a 2007. Revista Brasileira de Pesquisa em Turismo, 3 (03), 3-33.

Selltiz, C., Wrightsman, L. \& Cook, S. (1987). Métodos de pesquisa nas relações sociais. São Paulo: EPU.

Severino, A. J. (2007). Metodologia do trabalho científico. 23. ed. São Paulo: Cortez.

Silva, D., Lopes, E. L. \& Braga Junior, S. S. (2014). Pesquisa Quantitativa: Elementos, Paradigmas e Definições. Revista de Gestão e Secretariado, 5, 1-18. 
Silveira, C. E; Medaglia, J. \& Gândara, J. M G. (2012). Quatro décadas de ensino superior no Brasil: dificuldades na formações e consolidação do mercado de trabalho e a ascensão de uma área de estudo como efeito colateral. Revista Turismo Visão e Ação, 14 (1), 06-18.

Sogayar, R.L. \& Rejowski, M. (2011). Ensino superior em turismo em busca de novos paradigmas educacionais: problemas, desafios e forças de pressão. Turismo Visão e Ação,13 (03), 282-298.

Stumpf, I. R. C. (2010). Pesquisa Bibliográfica. In: Duarte, J.; Barros, A. (org.). Métodos e Técnicas de pesquisa em comunicação. São Paulo: Atlas.

Terence, A. C. F. \& Escrivão-Filho, E. (2006). Abordagem quantitativa, qualitativa e a utilização da pesquisa-ação nos estudos organizacionais. In: XXVI Encontro Nacional de Engenharia de Produção. Anais eletrônicos... Fortaleza: ENEGEP, 2006. Disponível em: http://www.abepro. org.br/biblioteca/ENEGEP2006_TR540368_8017.pdf. Acesso em 10 setembro 2016.

Teixeira, E. B. (2003). A análise de dados na pesquisa científica: importância e desafios em estudos organizacionais. Desenvolvimento em Questão, 1(2), 177-201.

Tribe, J. (2010). Tribes, Territories and Networks in Tourism Academy. Annals of Tourism Research, 37 (1), 7-33.

Tribe, J. \& Liburd, J. (2016). The tourism knowledge system. Annals of Tourism Research, 57, 44-61.

Veal, A.J. (2011). Metodologia de pesquisa em lazer e turismo. São Paulo: Aleph.

Vergara, S. C. (2005). Métodos de pesquisa em administração. São Paulo: Atlas.

Vergara, S. C. (2012). Métodos de coleta de dados no campo. 2. Ed. São Paulo: Atlas.

Yin, R. K. (2005). Estudo de caso: planejamento e métodos. 3. Ed. Porto Alegre: Bookman.

\section{CONTRIBUIÇÃO DOS AUTORES NA CONSTRUÇÃO DO ARTIGO}

COSTA: Coleta e processamento dos dados; Pesquisa para construção do texto; Elaboração do artigo; Revisão e colaboração na adequação do artigo para publicação.

TITO: Coleta e processamento dos dados; Pesquisa para construção do texto; Elaboração do artigo; Revisão e colaboração na adequação do artigo para publicação. 
BRUMATTI: Pesquisa para construção do texto; Elaboração do artigo; Revisão e colaboração na adequação do artigo para publicação.

ALEXANDRE: Orientação temática. 